What If the Heartbeat Counting Task Required No Measure of Cardiac Activity?

\author{
Olivier Desmedt ${ }^{1,2}$, Olivier Luminet ${ }^{1,2}$, Pierre Maurage $^{1} \&$ Olivier Corneille $^{1}$ \\ ${ }^{1}$ Psychological Science Research Institute, UCLouvain, Louvain-la-Neuve, Belgium \\ ${ }^{2}$ Fund for Scientific Research - Belgium (FRS-FNRS)
}

\begin{abstract}
Author Note
Olivier Desmedt is a FRESH grantee of the Fonds de la Recherche Scientifique - FNRS.

Correspondence concerning this article should be addressed to Olivier Desmedt, UCLouvainIPSY, 10 Place du Cardinal Mercier, B-1348 Louvain-la-Neuve, Belgium. Email:

olivier.desmedt@uclouvain.be.
\end{abstract}




\begin{abstract}
Heartbeat Counting Task (HCT) scores are thought to indicate people's objective ability to detect their cardiac signals (i.e., their cardiac interoceptive accuracy). In a re-analysis of a large sample of HCT scores, we found that these scores show a .80 correlation with the total number of reported heartbeats when using the original instructions allowing participants to estimate their heart rate, and a .97 correlation when using the (more valid) modified instructions that reduce the influence of estimation strategies. This extremely high correlation suggests that an objective measure of cardiac activity is hardly needed to establish HCT scores, at least when the measure is - as usually the case - collected at rest. This finding raises further important questions on the validity of the HCT as a measure of cardiac interoceptive accuracy, even when using the more valid modified instructions.
\end{abstract}

Keywords: interoception, interoceptive accuracy, heartbeat counting task. 


\section{What If the Heartbeat Counting Task Required No Measure of Cardiac Activity?}

Interoception is defined as the processing of inner body signals by the nervous system (Khalsa et al., 2017). One central dimension of interoception is the objective capacity to detect internal states (i.e., interoceptive accuracy; Garfinkel et al., 2015). This ability is thought to play a key role in mental and physical health (Ceunen et al., 2016; Khalsa et al., 2017). The most frequently used measure of interoceptive accuracy is the Heartbeat Counting Task (HCT; Dale \& Anderson, 1978; Schandry, 1981). The HCT has been widely used in psychology, psychiatry and neuroscience research because its non-intrusiveness would allow for a convenient and ethically acceptable investigation of interoceptive accuracy.

In the introduction, we briefly discuss the importance of disposing of a valid measure of interoceptive accuracy. We then present the HCT and discuss its limitations. In the empirical section, we analyze a large dataset of HCT scores collected under original and modified instructions. Based on the original findings reported here, we conclude that the HCT should not be used anymore as a measure of interoceptive accuracy, even when using the more valid modified instructions.

\section{Interoceptive accuracy and health}

Interoception is essential for survival as it informs the central nervous system about physiological needs (Craig, 2010; Damasio \& Carvalho, 2013; Gu et al., 2013) and thus initiates actions to attain or maintain homeostasis (Cannon, 1929). Interoception is also thought to play a key adaptative role in drives, feelings, emotional experiences, emotional regulation, and cognition (for a recent discussion, see Khalsa et al., 2017).

Humans, however, are not all equal in their ability to detect internal signals and this may be consequential for their mental and physical health. For instance, individuals with low interoceptive abilities have been found to present more frequent dysfunctional behaviors (e.g., 
addiction; Verdejo-Garcia et al., 2012), mental disorders (e.g., depression; Dunn et al., 2007), and physical health issues (e.g., obesity; Herbert \& Pollatos, 2014).

Some authors have proposed that interoceptive accuracy could be a transdiagnostic process (i.e., a hub process) involved in multiple disorders (Khalsa et al., 2017). As a result, interventions targeting the improvement of interoceptive accuracy could have a positive impact across many pathological states. More generally, interoceptive accuracy may constitute a core process in a large range of cognitive (e.g., decision-making; Dunn et al., 2010), behavioral (e.g., consumption behavior; Herbert et al., 2013) and emotional (e.g., emotional regulation; Füstös et al., 2013; but see Zamariola et al., 2019) processes.

\section{A convenient measure of interoceptive accuracy: the HCT.}

As it appears, a valid measure of interoceptive accuracy is critical to theory and practice. Unfortunately, performance measures of interoception are undermined by important methodological limitations. In particular, hundreds of studies have relied on the HCT (Dale \& Anderson, 1978; Schandry, 1981) to assess interoceptive accuracy. In this task, participants at rest are instructed to count how many times their heart is beating during short time intervals. Their self-report is then compared to their objective heartbeats. HCT scores are established by computing the following formula: mean of $(1-(\mid$ actual heartbeats - reported heartbeats $\mid$ )/actual heartbeats). The smaller the absolute difference between their reported and objective number of heartbeats, the higher an HCT score and the higher a participant's level of interoceptive accuracy.

The HCT provides a convenient (i.e., non-intrusive, non-expensive) measure of interoceptive accuracy. It also has face validity: intuitively, the better people are able to match their reported heartbeats on their objective cardiac activity, and the better their interoceptive accuracy should be. However, critiques have accumulated regarding the validity of this task (for a recent discussion, see Corneille et al., 2020). One major problem with this task is that 
participants can use their prior knowledge for completing the task. Therefore, estimation rather than experiential (i.e., interoceptive) processes can affect task performance.

The role of estimation processes is particularly evident in the vast majority of studies that relied on original instructions (e.g., Ring et al., 2015; Ring \& Brener, 2018) that leave room for estimation strategies in task completion. A recent study found that, when using modified instructions asking participants to only report their felt heartbeats (without relying on estimation processes), HCT scores are reduced by 50\% (Desmedt et al., 2018). Another recent study also indicates correlations between reported heartbeats and estimation strategies are higher under original than modified instructions (Desmedt, Corneille, et al., 2020). These findings indicate that estimation processes largely contribute to HCT scores when using the original instructions, and that modified instructions should therefore be preferred.

That the role of estimation processes is largely reduced under modified instructions, however, still does not guarantee that the HCT allows for a valid measure of interoceptive accuracy. Rather, it guarantees that the influence of estimation processes is reduced when using the modified instructions (Desmedt, Corneille, et al., 2020).

As a matter of fact, a second important limitation plaguing the interpretation of HCT scores is that the task structure does not allow distinguishing between sensitivity (i.e., the capacity to tease apart cardiac signal and noise) and decision threshold (i.e., the willingness to report a cardiac signal; Desmedt, Dekeyser, et al., 2020). This is due to a lack of experimental control over the cardiac signal (see Corneille et al., 2020; Zamariola et al., 2018).

A third important issue is that HCT scores mostly reflect the extent to which participants underestimate their actual heartbeats. That is, only a few respondents report more heartbeats than those objectively recorded. Some authors have argued that this is not so problematic because the measure is not conceptually concerned with "hallucinatory" perception, but is rather concerned with respondents' ability to access their (true) cardiac 
activity (Ainley et al. 2020). We have explained elsewhere why we believe this point is problematic (Corneille et al. 2020). Yet, we can agree it is a matter of theoretical preference. What would be hardly defensible, however, is if HCT scores essentially reflect one of their two measured components only (i.e., the objective or the subjective component). This is because the HCT is conceptually concerned with the discrepancy between the two, which is what accuracy is about.

In the present research, we re-analyzed HCT scores under modified instructions by correlating these scores with reported heartbeats. We reasoned that, if HCT scores truly reflect interoceptive accuracy, then this correlation should be modest. That is, a measure meant to reflect a discrepancy between reported and objective heartbeats should not be redundant with its subjective component only. For the sake of completeness, we additionally report the analyses for scores obtained under original instructions, although we have explained above why the original instructions lack validity.

\section{Method}

Data were borrowed from Zamariola et al. (Study1; N=572; 2018) ${ }^{1}$, Desmedt et al. (Study2; N=121; 2018) ${ }^{2}$ and Desmedt, Dekeyser et al. (Study 3; N=164; 2020) ${ }^{3}$. Participants were healthy students at the UCLouvain, recruited through advertisements on a Facebook page, mailing lists or at the Faculty. They were all compensated for their participation. Ethical approval was granted by the local ethics committee. Materials, procedures and data handling strategies (i.e., data exclusion and missing data) are available in the original articles.

Different instructions were used when administering the HCT: original instructions (Desmedt et al., 2018; Zamariola et al., 2018): “Count your heartbeats without touching your wrists or neck pulsations"; modified instructions (Desmedt et al., 2018): "It is very important

\footnotetext{
${ }^{1}$ Data will be available upon submission at the following address: XXX.

${ }^{2}$ Data will be available upon submission at the following address: XXX.

${ }^{3}$ Data will be available upon submission at the following address: XXX.
} 
that you only count the heartbeats you really feel, without trying to guess your heart rate"; modified conservative instructions (Desmedt, Dekeyser, et al., 2020): "count a heartbeat only when you are very confident that you have really perceived one"; modified liberal instructions: "count a heartbeat even when you are relatively unconfident that you have perceived one". While original instructions are problematic, as they implicitly allow participants to estimate their heart rate, all modified instructions efficiently reduce the use of estimation strategies.

\section{Results}

HCT scores were computed using the classic formula: $1 / 3 \Sigma(1-(\mid$ actual heartbeats reported heartbeats|)/actual heartbeats), with higher scores indicating higher accuracy. The raw correlations ${ }^{4}$ between HCT scores and reported heartbeats are reported on the first line of Table 1 (see also Figure 1 for the scatterplot). For the sake of completeness, we also report in Table 1 (second line) the correlations between HCT scores and objective heartbeats. ${ }^{5}$

\section{Table 1}

Pearson correlations of HCT scores with reported heartbeats (line 1), and actual heartbeats

(line 2), for studies involving original and modified instructions

\begin{tabular}{|c|c|c|c|c|c|}
\hline & Study 1 & Study 2 & Study 2 & Study 3 & Study 3 \\
\hline & Original & Original & Modified & Conservative & Liberal \\
\hline Reported heartbeats & $.80 * *$ & $.80 * *$ & $.97 * *$ & $.97 * *$ & $.96 * *$ \\
\hline Actual heartbeats & $-.35 * *$ & $-.31 * *$ & $-.21 *$ & -.07 & -.13 \\
\hline
\end{tabular}

\footnotetext{
${ }^{4}$ Very similar results are found when using non-parametric analyses (i.e., Spearman correlations). ${ }^{5}$ Very similar results are found when excluding participants with extreme heart rates and extreme heart rate differences between time intervals (i.e., heart rate and mean difference of heart rate between intervals of more than three times the interquartile range). These values may indicate reliability issues with the heart monitor devices.
} 
**. Correlation is significant at the .01 level (2-tailed).

\section{Figure 1}

Scatterplot of associations between HCT scores and reported heartbeats under original and modified instructions (Desmedt et al., 2018)

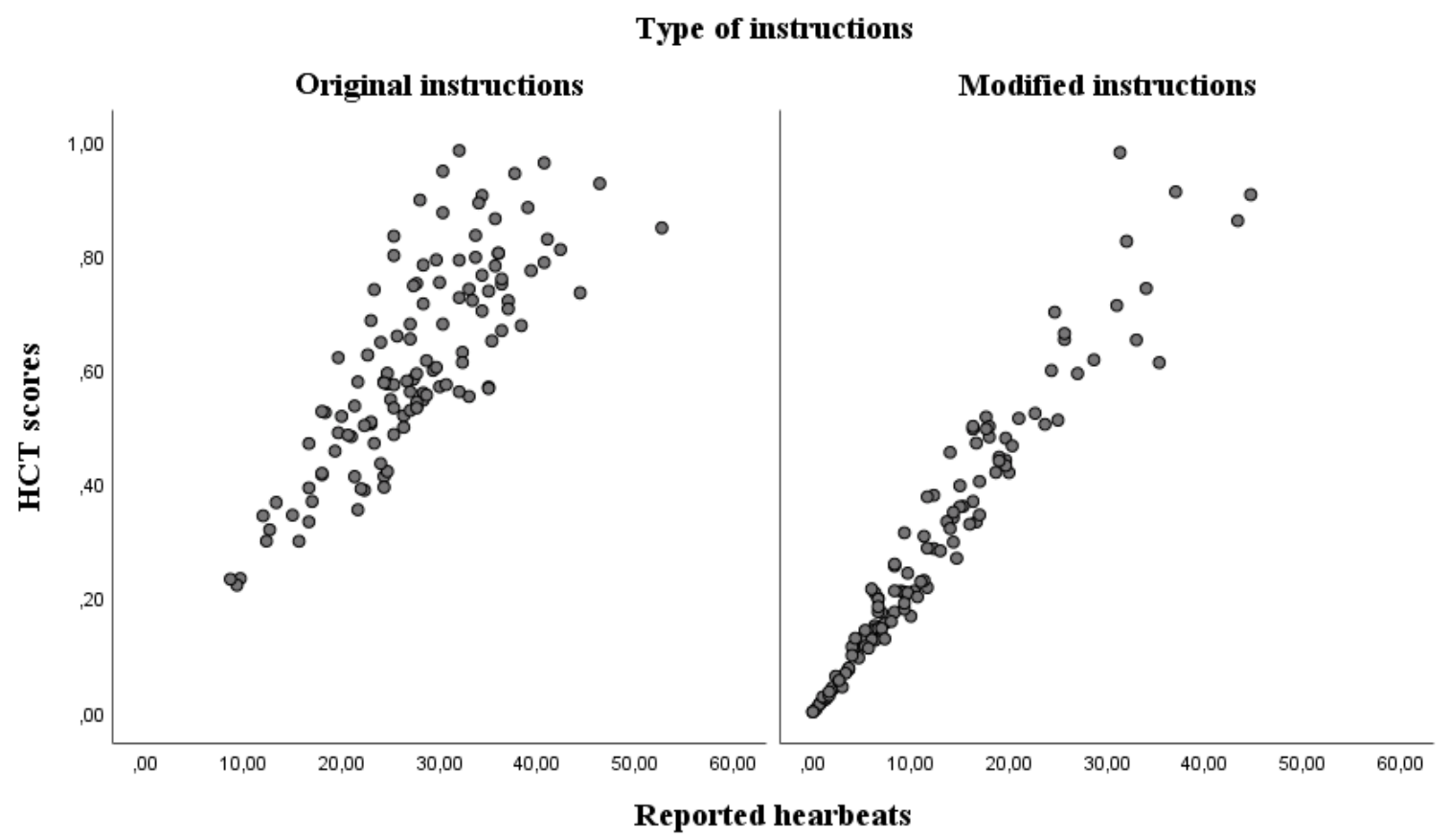

\section{Discussion}

The implication of the present findings is straightforward: under modified (i.e., more valid) instructions, HCT scores can be almost perfectly predicted without actually recording participants' objective cardiac activity. This may point to a convenient adaptation of the procedure: because cardiac recording is hardly needed for establishing HCT scores, the HCT may be adapted by removing cardiac recording and by merely considering reported heartbeats as a measure of interoceptive accuracy. The problem with this recommendation should be self-evident when the task is meant to assess people's sensitivity to cardiac signals. For the sake of completeness, we also tested the correlation between HCT scores and objective heartbeats. How this correlation should be interpreted, and how problematic it is, has been discussed elsewhere (Corneille et al., 2020; Zimprich et al., 2020). In any case, this 
correlation appears to be way smaller than the near-perfect association found between HCT scores and reported heartbeats.

We believe that, because the task is too difficult to complete at rest, participants cannot reach the target (i.e., sense their heartbeats), which makes the target (i.e., objective heartbeats) useless. It would be interesting to examine in future research how the correlation is affected when using modified instructions under conditions of physiological activation (see Smith et al., 2020). The validity of the task may increase under such conditions. That is, as the heartbeat signal becomes stronger, the task may become easier and so it may also become more sensitive than when the task is performed at rest (Schandry et al., 1993). This being said, given the absence of control over the heartbeat signal, the task would still not allow teasing apart sensitivity and decision threshold, making it a poor measure of interoceptive accuracy (see Corneille et al., 2020).

A narrow interpretation of the current findings may point to a psychometric necessity. Specifically, because most HCT scores reflect an underestimation (see Zamariola et al., 2018), a large correlation is to be expected between HCT scores and reported heartbeats if heart rates show little variation across participants. There are three points to be considered here. First, there actually was a fair amount of variability in actual heartbeats in our samples (e.g., in Study 2 - modified instructions - they ranged, on average, from 29 to 59 heartbeats per interval). Second, no matter the psychometric necessity, it would still be the case that objective recording is hardly needed for establishing HCT scores. Third, the lack of variability may precisely be due to the fact that the task is completed at rest. As we noted above, cardiac signals may not be sufficiently intense at rest to allow for a valid estimation of cardiac interoceptive accuracy, and heart rate may not be sufficiently variable either at rest for avoiding the sort of psychometric implications we discuss here. 
Because HCT scores hardly require an objective measure of cardiac activity when collected at rest, we believe it is fair to conclude that studies that relied on these scores did not make use of a valid measure of interoceptive accuracy. Instead, these studies essentially measured the extent to which participants report heartbeats (i.e., interoceptive reporting). As provocative as it may seem, these studies essentially captured another subjective, selfreported, measure of interoception. As explained in Corneille et al. (2020), a host of factors may underly such reporting, including a collection of non-interoceptive processes and interoceptive processes, the latter of which may or may not be related to accuracy.

By demonstrating that a measure that has widely been considered a measure of interoceptive accuracy is essentially a subjective measure of interoception, the present research goes well beyond current critiques communicated about this task. That an objective measure of cardiac activity is not necessary for almost perfectly approximating HCT scores, we believe is the ultimate demonstration that, when collected at rest, HCT scores can hardly be considered a valid measure of cardiac interoceptive accuracy.

Whether it represents a valid measure of something else may be clarified in future studies. 


\section{Acknowledgments and Funding Information}

Olivier Desmedt (PhD student), Olivier Luminet (Research Director) and Pierre Maurage (Senior Research Associate) are funded by the Fund for Scientific Research - Belgium (FRSFNRS). 


\section{References}

Ainley, V., Tsakiris, M., Pollatos, O., Schulz, A., \& Herbert, B. M. (2020). Comment on “Zamariola et al. (2018), Interoceptive Accuracy Scores are Problematic: Evidence from Simple Bivariate Correlations"-The empirical data base, the conceptual reasoning and the analysis behind this statement are misconceived and do not support the authors' conclusions. Biological Psychology, 152, 107870. https://doi.org/10.1016/j.biopsycho.2020.107870

Cannon, W. B. (1929). Organization for physiological homeostasis. Physiological Reviews, 9(3), 399-431. https://doi.org/10.1152/physrev.1929.9.3.399

Ceunen, E., Vlaeyen, J. W., \& Van Diest, I. (2016). On the origin of interoception. Frontiers in Psychology, 7, 743. https://doi.org/10.3389/fpsyg.2016.00743

Corneille, O., Desmedt, O., Zamariola, G., Luminet, O., \& Maurage, P. (2020). A Heartfelt Response to Zimprich et al.(2019), and Ainley et al.(2019)'s Commentaries: Acknowledging issues with the HCT would Benefit Interoception Research. Biological Psychology, 107869. https://doi.org/10.1016/j.biopsycho.2020.107869

Craig, A. D. (2010). The sentient self. Brain Structure and Function, 214(5-6), 563-577. https://doi.org/10.1007/s00429-010-0248-y

Dale, A., \& Anderson, D. (1978). Information variables in voluntary control and classical conditioning of heart rate: Field dependence and heart-rate perception. Perceptual and Motor Skills, 47(1), 79-85. https://doi.org/10.2466/pms.1978.47.1.79

Damasio, A. R., \& Carvalho, G. B. (2013). The nature of feelings: Evolutionary and neurobiological origins. Nature Reviews Neuroscience, 14(2), 143. https://doi.org/10.1038/nrn3403

Desmedt, O., Corneille, O., Luminet, O., Murphy, J., Bird, G., \& Maurage, P. (2020). Contribution of Time Estimation and Knowledge to Heartbeat Counting Task 
Performance under Original and Adapted Instructions. Biological Psychology, 107904. https://doi.org/10.1016/j.biopsycho.2020.107904

Desmedt, O., Dekeyser, S., Luminet, O., \& Corneille, O. (2020). Investigating the link between Interoceptive accuracy, Alexithymia, and Trait Anxiety under Adapted Heartbeat Counting Task Instructions. Manuscript in preparation.

Desmedt, O., Luminet, O., \& Corneille, O. (2018). The heartbeat counting task largely involves non-interoceptive processes: Evidence from both the original and an adapted counting task. Biological Psychology, 138, 185-188.

https://doi.org/10.1016/j.biopsycho.2018.09.004

Dunn, B. D., Dalgleish, T., Ogilvie, A. D., \& Lawrence, A. D. (2007). Heartbeat perception in depression. Behaviour Research and Therapy, 45(8), 1921-1930. https://doi.org/10.1016/j.brat.2006.09.008

Dunn, B. D., Galton, H. C., Morgan, R., Evans, D., Oliver, C., Meyer, M., Cusack, R., Lawrence, A. D., \& Dalgleish, T. (2010). Listening to your heart how interoception shapes emotion experience and intuitive decision making. Psychological Science, 21(12), 1835-1844. https://doi.org/10.1177/0956797610389191

Füstös, J., Gramann, K., Herbert, B. M., \& Pollatos, O. (2013). On the embodiment of emotion regulation: Interoceptive awareness facilitates reappraisal. Social Cognitive and Affective Neuroscience, 8(8), 911-917. https://doi.org/10.1093/scan/nss089

Garfinkel, S. N., Seth, A. K., Barrett, A. B., Suzuki, K., \& Critchley, H. D. (2015). Knowing your own heart: Distinguishing interoceptive accuracy from interoceptive awareness. Biological Psychology, 104, 65-74. https://doi.org/10.1016/j.biopsycho.2014.11.004

Gu, X., Hof, P. R., Friston, K. J., \& Fan, J. (2013). Anterior insular cortex and emotional awareness. Journal of Comparative Neurology, 521(15), 3371-3388.

https://doi.org/10.1002/cne.23368 
Herbert, B. M., Blechert, J., Hautzinger, M., Matthias, E., \& Herbert, C. (2013). Intuitive eating is associated with interoceptive sensitivity. Effects on body mass index. Appetite, 70, 22-30. https://doi.org/10.1016/j.appet.2013.06.082

Herbert, B. M., \& Pollatos, O. (2014). Attenuated interoceptive sensitivity in overweight and obese individuals. Eating Behaviors, 15(3), 445-448. https://doi.org/10.1016/j.eatbeh.2014.06.002

Khalsa, S. S., Adolphs, R., Cameron, O. G., Critchley, H. D., Davenport, P. W., Feinstein, J. S., Feusner, J. D., Garfinkel, S. N., Lane, R. D., \& Mehling, W. E. (2017). Interoception and mental health: A roadmap. Biological Psychiatry: Cognitive Neuroscience and Neuroimaging, 3, 501-503. https://doi.org/10.1016/j.bpsc.2017.12.004

Ring, C., \& Brener, J. (2018). Heartbeat counting is unrelated to heartbeat detection: A comparison of methods to quantify interoception. Psychophysiology, e13084. https://doi.org/10.1111/psyp.13084

Ring, C., Brener, J., Knapp, K., \& Mailloux, J. (2015). Effects of heartbeat feedback on beliefs about heart rate and heartbeat counting: A cautionary tale about interoceptive awareness. Biological Psychology, 104, 193-198. https://doi.org/10.1016/j.biopsycho.2014.12.010

Schandry, R. (1981). Heart beat perception and emotional experience. Psychophysiology, 18(4), 483-488. https://doi.org/10.1111/j.1469-8986.1981.tb02486.x

Schandry, R., Bestler, M., \& Montoya, P. (1993). On the relation between cardiodynamics and heartbeat perception. Psychophysiology, 30(5), 467-474. https://doi.org/10.1111/j.1469-8986.1993.tb02070.x

Smith, R., Feinstein, J., Kuplicki, R., Forthman, K. L., Stewart, J. L., Paulus, M., Khalsa, S. S., \& Investigators, T. (2020). Perceptual insensitivity to the modulation of 
interoceptive signals in depression, anxiety, and substance use disorders. Manuscript submitted for publication.

Verdejo-Garcia, A., Clark, L., \& Dunn, B. D. (2012). The role of interoception in addiction: A critical review. Neuroscience \& Biobehavioral Reviews, 36(8), 1857-1869. https://doi.org/10.1016/j.neubiorev.2012.05.007

Zamariola, G., Luminet, O., Mierop, A., \& Corneille, O. (2019). Does it help to feel your body? Evidence is inconclusive that interoceptive accuracy and sensibility help cope with negative experiences. Cognition and Emotion, 1-12. https://doi.org/10.1080/02699931.2019.1591345

Zamariola, G., Maurage, P., Luminet, O., \& Corneille, O. (2018). Interoceptive accuracy scores from the heartbeat counting task are problematic: Evidence from simple bivariate correlations. Biological Psychology, 137, 12-17. https://doi.org/10.1016/j.biopsycho.2018.06.006

Zimprich, D., Nusser, L., \& Pollatos, O. (2020). Are interoceptive accuracy scores from the heartbeat counting task problematic? A comment on Zamariola et al. (2018). Biological Psychology, 152, 107868. https://doi.org/10.1016/j.biopsycho.2020.107868 